\title{
Impact des aménagements pour la navigation sur les niveaux d'eau de la Seine en aval de Paris
}

\author{
par P. de Guibert
}

Service de la Navigation de la Seine

\section{I $\square$ LA SEINE EN AVAL DE PARIS AVANT 1910}

Jusqu'au début du XIXème siècle, la navigation sur la Seine s'est effectuée dans de mauvaises conditions : d'une part elle était interrompue près de six mois par an en raison principalement des basses eaux, d'autre part, le chenal comportait de nombreux passages difficiles, constitués en particulier par des pertuis étroits à l'emplacement d'anciens ponts mal construits.

La première canalisation de la Seine remonte à 1840 , et fut envisagée à la suite de l'invention par Poirée de barrages à fermettes mobiles et à aiguilles dont l'application permit d'assurer sur la rivière un mouillage (profondeur) de 1,60 m par la construction de cinq biefs : Bezons-Bougival (1840) ; Andrésy-Denouval (1846) - Les Mureaux (1853) ; NotreDame de la Garenne (1847) ; Poses (1850) (figure 1).

A peine cette réalisation était-elle terminée, qu'on décidait de porter le mouillage à $2 \mathrm{~m}$ en exhaussant les barrages existants et en en créant trois nouveaux entre 1859 et 1866 à Martot, Villez et Suresnes. Tous les barrages construits étaient du type à fermettes, et comportaient une écluse accolée ayant en principe $113 \mathrm{~m}$ de long, $12 \mathrm{~m}$ de large.

Mais la situation qui prévalait en 1910 remonte à 1880 (programme Freycinet) : mouillage porté à $3,20 \mathrm{~m}$ par exhaussement des retenues d'eau. Pour cela on avait dû reconstruire quatre barrages et en construire un neuvième à Méricourt-Sandrancourt. C'est à cette époque qu'apparurent sur la Seine les barrages à cadres Caméré et le groupe de deux écluses (une grande et une petite) établi à chaque chute.

Ces premiers travaux de canalisation de la rivère n'avaient que très peu d'incidences sur les conditions d'écoulement du fleuve. En effet, l'augmentation du mouillage était essentiellement obtenue par exhaussement artificiel du niveau de la rivière pour les basses eaux ; au-delà d'un certain débit (débit des plus basses eaux navigables), les barrages de navigation étaient entièrement effacés, et le mouillage naturel supérieur à $3,20 \mathrm{~m}$. Seuls quelques seuils avaient dû être supprimés, quelques ponts reconstruits.

\section{II $\square$ LES PROJETS ET LES AMÉNAGEMENTS APRÈS 1910}

\subsection{Le programme Perrier}

Après un premier programme d'aménagement d'ensemble de la Basse Seine présenté par Vidal en 1918, Perrier en proposa en 1921 un nouveau qui fit l'objet de la décision ministérielle du 10 août 1922 et qui avait un triple objectif :

a) L'amélioration des conditions de navigation, justifiée par l'augmentation importante et constante des trafics :

- par la suppression de trois biefs ramenant leur nombre à six ;

- par l'augmentation du mouillage porté en première phase à $3,50 \mathrm{~m}$, à $5 \mathrm{~m}$ en deuxième phase ;

- par la réalisation d'un chenal de $80 \mathrm{~m}$ de largeur minimum ;

- par la construction à chaque barrage d'une nouvelle écluse de $275 \mathrm{~m}$ par $17 \mathrm{~m}$.

b) L'amélioration des conditions d'écoulement des grandes crues, par l'approfondissement du lit pour obtenir un abaissement général du plan d'eau en période de crue, et son calibrage pour offrir en tout point un débouché suffisant à l'écoulement des eaux.

c) L'utilisation de l'énergie hydraulique des chutes par la construction d'une usine hydro-électrique à chaque barrage.

Ce programme prévoyait notamment le dragage de $32 \mathrm{mil}-$ lions de $\mathrm{m}^{3}$ de matériaux et des travaux de défense des

The fitting up of down-stream Seine for sailing has a limited impact on the flood draining off. But a fitting up of the river Oise, which is planned for the future large-size Seine-Nord binding, would have a more important impact in the case of a flood. 


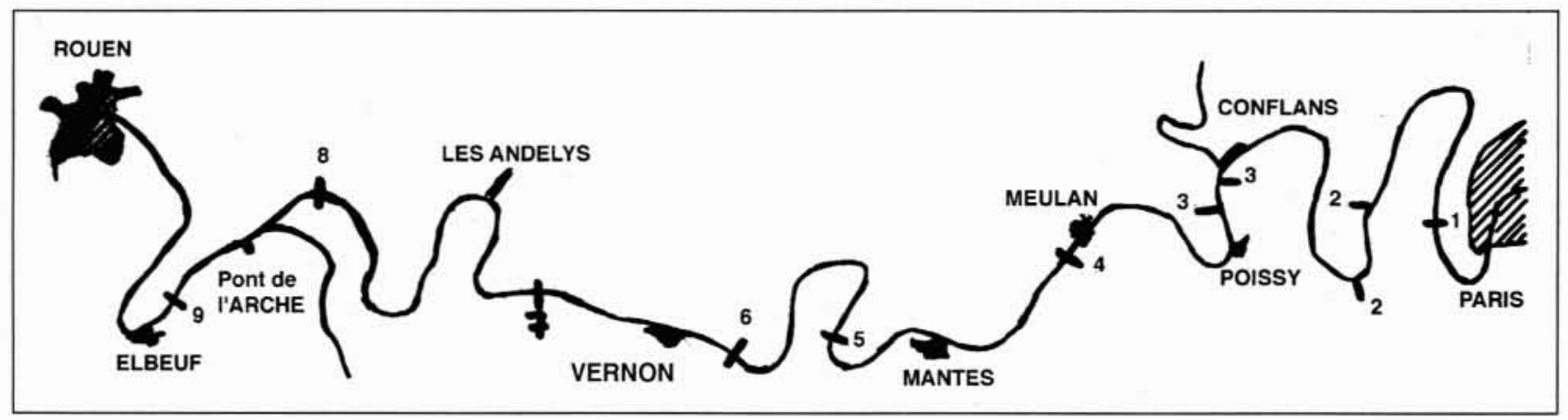

1. Les aménagements en aval de Paris vers 1910.
1. Suresnes
6. Villez
2. Chatou-Bougival
7. Notre-Dame de la Grande
3. Andrésy-Denouval
8. Poses-Amfreville
4. Les Mureaux
9. Martot

5. Méricourt-Sandrancourt

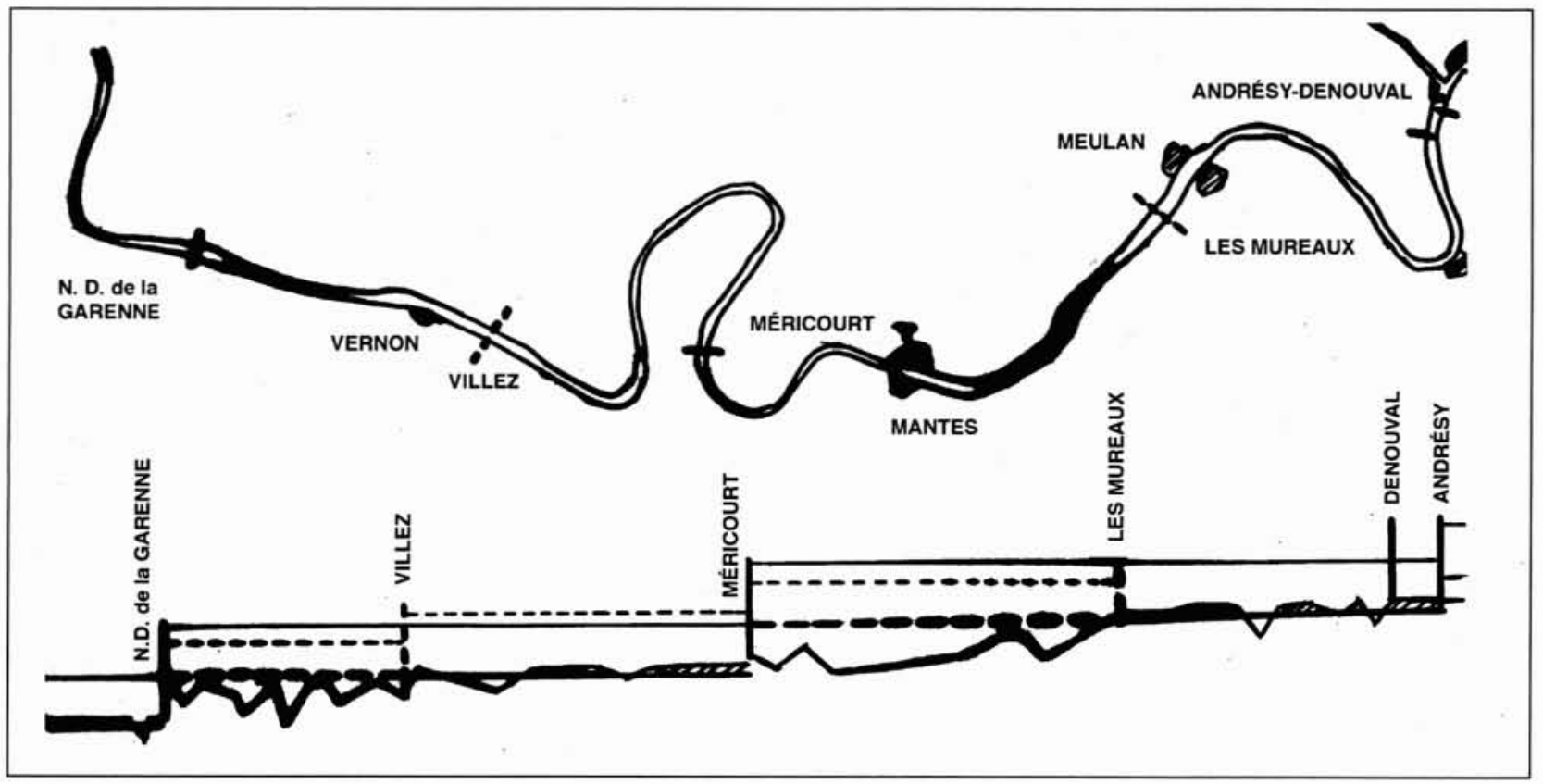

2. Profil de la Seine : suppression des barrages des Mureaux et de Villez.

berges (calibrage) correspondant pour l'essentiel à l'objectif d'amélioration des conditions d'écoulement de la rivière. Un calcul rapide et très sommaire montre qu'en moyenne la section d'écoulement avant débordement de la rivière aurait été ainsi augmentée de $160 \mathrm{~m}^{2}$, soit d'environ $13 \%$. Les crues non débordantes auraient été abaissées d'environ $60 \mathrm{~cm}$, la crue de type 1910 de quelques dizaines de $\mathrm{cm}$ au plus.

De 1932 à 1939, ce programme a reçu un début de réalisation : suppression du bief de Martot, reconstruction des barrages d'Andrésy et de Chatou, construction d'une nouvelle écluse et approfondissement d'une autre à Poses. Mais les travaux d'amélioration des conditions de l'écoulement n'ont pas été engagés.

Après 1946, un grand nombre de ponts détruits pendant la guerre ont été reconstruits avec un tirant d'air de $7 \mathrm{~m}$ au dessus des Plus Hautes Eaux Navigables (PHEN).

\section{- 2.2 Le programme des années 1960}

A la fin des années 1950 la Basse-Seine canalisée comportait encore huit biefs jusqu'à Paris. La situation avait en fait très peu évolué depuis 1910 (à l'exception de quelques barrages de navigation reconstruits et de quelques nouvelles écluses plus grandes) : tirant d'eau autorisé de $3 \mathrm{~m}$ pour un chenal d'une profondeur minimum de $3,20 \mathrm{~m}$ par rapport au niveau de la retenue normale et, sauf quelques exceptions, d'une largeur supérieure à $50 \mathrm{~m}$, celle-ci étant le plus souvent comprise naturellement entre 80 et $100 \mathrm{~m}$.

L'augmentation très importante du trafic $(3,5$ millions de tonnes en 1900, 7 millions en 1950, 12 millions en 1960), ses perspectives d'évolution (en 1968 le trafic atteindra 22 millions de tonnes), le développement de la navigation par convois poussés, conduisent alors à engager et réaliser totalement à partir de 1959 un important programme d'améliora- 
tion de la canalisation afin d'augmenter sa capacité de transport :

- construction d'écluses au mouillage de $5 \mathrm{~m}$, de $160 \mathrm{~m}$ de long, puis de $185 \mathrm{~m}$ de long,

- suppression de deux biefs ( suppression des barrages des Mureaux et de Villez ),

- augmentation du mouillage du chenal à $4 \mathrm{~m}$,

- reconstruction-modernisation des barrages anciens,

- reconstruction des ponts en aval de Gennevilliers ne dégageant pas $7 \mathrm{~m}$ de tirant d'air au dessus des PHEN.

L'objectif d'amélioration des conditions d'écoulement de la rivière envisagé par le programme Perrier n'était pas repris.

L'augmentation du mouillage du chenal de navigation était obtenu soit par rehaussement de certains plans d'eau, dans le cas des fusions de bief, soit par dragages (figure 2).

Dans le cas des fusions de bief (suppression des barrages des Mureaux et de Villez), les plans d'eau tenus par les barrages de Notre-Dame de la Garenne et de Méricourt étaient rehaussés, ceci nécessitant la réalisation d'endiguements en amont immédiat de ces deux barrages, surtout dans le cas de Méricourt.

Ailleurs le chenal était approfondi de 80 centimètres par dragages.

Dans tous les cas on avait cherché à limiter les dragages nécessaires pour des raisons de coût d'investissement, mais également d'entretien ultérieur.

L'incidence de ces divers travaux sur les niveaux de crue est difficile à évaluer. Elle résulte du cumul de la suppression de pertes de charge localisées (reconstruction de certains ponts), des dragages et aux approches de certaines écluses de recalibrages de courbes très limités. Au total cette incidence ne doit pas excéder la dizaine de $\mathrm{cm}$ pour les crues moyennes.

A noter que les campagnes annuelles de dragage d'entretien imposées par le maintien de la navigation, permettent de garantir une section d'écoulement constante pour la rivière.

\section{III $\square$ CONCLUSION}

L'aménagement pour la navigation de la Seine en aval de Paris n'a qu'une faible incidence sur les niveaux de crue de la rivière. Les dimensions naturelles de la rivière sont telles qu'un chenal de navigation d'au moins $50 \mathrm{~m}$ de large et $4 \mathrm{~m}$ de profondeur a été réalisé sans rectifications importantes de son lit.

Il n'en est pas de même pour une rivière plus modeste.

Cela peut être illustré par le projet d'achèvement de la mise à grand gabarit de l'Oise de Compiègne jusqu'à la Seine, projet qui vise des caractéristiques pour la navigation proches de celles existant aujourd'hui sur la Basse-Seine, notamment un mouillage de $4 \mathrm{~m}$.

Toutefois, compte tenu du débit et des dimensions plus modestes de la rivière Oise, la mise à grand gabarit nécessite des rectifications de courbes et un approfondissement subtantiel de son lit. Ce projet a été très partiellement réalisé au début des années 1970. Les études relancées en 1995 par Voies Navigables de France dans le cadre du projet de liaison Seine-Nord doivent permettre d'analyser l'ensemble des impacts résultant de l'aménagement, notamment l'impact sur le fonctionnement hydraulique de la rivière. C'est pourquoi une modélisation de ce fonctionnement a été réalisée, en poursuivant des travaux déjà engagés par l'Entente interdépartementale Oise-Aisne.

Le projet ne prévoit pas de modifier le nombre de biefs sur la rivière, ni le niveau de retenue de ceux-ci, mais de rectifier et d'approfondir le chenal de navigation.

Aujourd'hui l'analyse de l'impact d'une première ébauche de tracé est en cours. Les premiers résultats, partiels, mettent en évidence un abaissement conséquent des niveaux de crue : plus de 50 centimètres à proximité de Compiègne, valeur s'atténuant progressivement jusqu'à zéro à la confluence de la Seine. Ces résultats, incomplets, ne sont pour l'instant pas validés, pas plus que l'ébauche de tracé sur laquelle ils sont fondés qui doit faire l'objet très prochainement d'une démarche de concertation intégrée aux études auprès des collectivités et acteurs locaux concernés. 